\title{
Comparison of exercise therapies across multiple tendinopathies: A systematic review and network meta-analysis protocol.
}

Swinton, P.A. ${ }^{*}$ Shim, J. ${ }^{1}$ Pavlova, A.V. ${ }^{1}$ Moss, R. A. ${ }^{1}$ Maclean, C. ${ }^{2}$ Brandie, D. ${ }^{3}$ Mitchell, L. ${ }^{4}$ Greig, L. ${ }^{1}$ Parkinson, E. ${ }^{1}$ Morrissey, D. ${ }^{5}$ Alexander, L. ${ }^{1}$ Cooper, K. ${ }^{1}$

\section{DOI: $10.31236 /$ osf.io/eyxgk}

SportR $\chi$ iv hosted preprint version 1

Last updated

PREPRINT - NOT PEER REVIEWED

Institutions

${ }^{1}$ School of Health Sciences, Robert Gordon University, Aberdeen, UK

2 Library Services, Robert Gordon University, Aberdeen, UK

${ }^{3}$ Sport Scotland, Inverness, UK

${ }^{4}$ NHS Grampian, Aberdeen, UK

${ }^{5}$ Centre for Sports \& Exercise Medicine, Barts \& the London School of Medicine \& Dentistry, London, UK

* Corresponding Author

Dr. Paul Swinton

School of Health Sciences, Robert Gordon University

Garthdee Road

Aberdeen, UK,

AB10 7QG

p.swinton@,rgu.ac.uk, +44 (0) 12242623361

\section{Funding}

This project is funded by the National Institute for Health Research (NIHR) [Health Technology Assessment (HTA) 129388 Exercise therapy for the treatment of tendinopathies]. The views expressed are those of the authors and not necessarily those of the NIHR or the Department of Health and Social Care.

\section{Conflicts of interest}

The authors declare no conflict of interest. 


\subsection{Introduction}

Tendinopathy is a common musculoskeletal condition associated with degenerative changes within a tendon affecting both athletic and non-athletic populations. ${ }^{1}$ The condition is characterized by a combination of pain, ${ }^{1}$ and impaired movement ${ }^{2}$ and function ${ }^{3}$, requiring extended periods for recovery. ${ }^{2,4-5}$ Tendinopathy can affect any muscle-tendon unit in the body, ${ }^{6}$ however, it is most frequently reported in the Achilles, patellar, lateral elbow, rotator cuff, and hip tendons. ${ }^{6}$ Surveys of prevalence of lower extremity tendinopathy in the general population have reported rates of 11.8 and 10.5 per 1000 person-years, ${ }^{7}$ whilst prevalence for upper limb tendinopathies have been estimated between 1.3\% to 21.0\%. ${ }^{8-10}$ Tendinopathies can affect children, adolescents, and adults of all ages, and many tendinopathies have a chronic or recurrent course. ${ }^{6}$ Costs to the individual, the health service and economy (due to absenteeism and loss of productivity) are substantial such that identifying effective interventions is a priority. Musculoskeletal conditions including tendinopathies also have a substantive influence on primary and secondary healthcare use. ${ }^{11}$ By identifying effective interventions across a range of tendinopathies, General Practitioners and other first-contact practitioners (e.g. physiotherapists) can be confident in delivering effective evidence-based practice. With an ageing population, and increasing pressure and demands on healthcare services, the need for clear guidance for evidence-based practice has never been more important.

Exercise therapy is the mainstay of conservative management of tendinopathy and has focused largely on resistance training, and in many instances eccentric strengthening techniques, to date. ${ }^{12}$ The objective with exercise therapy is to encourage load tolerance that leads to structural adaptation at the musculotendinous unit and restores function. ${ }^{13-14}$ Isometric, isotonic, and heavy slow resistance training have also been recommended for some tendinopathies (e.g. patellar) with suggested efficacy. ${ }^{15}$ In the early phase of rehabilitation, range of movement and flexibility exercises are often initiated and incorporated into strengthening regimes to facilitate improvements in mobility. ${ }^{12}$ Included exercises range from static stretches to ballistic actions and variations of contract-relax stretching adapted from the proprioceptive neuromuscular facilitation literature. ${ }^{12}$ Effective exercise therapy may also require targeting a range of contributing factors, which not only include muscle weakness and decreased flexibility, but also corticospinal and neuromuscular adaptations resulting from persistent pain. ${ }^{16}$ As such, movement retraining or motor control-based exercise interventions have been used to retrain normal patterns of muscle recruitment in the rehabilitation of shoulder-related tendinopathies including impingement, with supportive evidence provided in trials and systematic reviews. ${ }^{16-19}$ Similarly, balance and core stabilisation exercises have been recommended for patients presenting with lumbo-pelvic instability in conjunction with patellar and Achilles tendinopathy. ${ }^{20}$ Whilst various exercise therapies have been proposed for the treatment of tendinopathy and the overarching aims of reducing pain and disability, and improving function, recommendations are frequently equivocal with no consensus on treatment guidelines for major tendinopathies.

Several previous systematic reviews have compared the effectiveness of different exercise therapies, with comparisons investigating exercise specificity (e.g. general vs specific exercises), ${ }^{21}$ exercise setting (supervised vs home), ${ }^{22}$ contraction mode (e.g. eccentric, concentric or isometric), ${ }^{23}$ and application of progressive overload (e.g. progressive vs non-progressive resisted exercise $)^{24}$. While some systematic reviews have provided evidence 
of differentiation, ${ }^{25,26}$ many have suggested there is equivalence between approaches ${ }^{21-24}$ and questioned the validity of entrenched focus on certain exercise protocols. ${ }^{26}$ Previous reviews comparing exercise therapies have generally been consistent in their overall approach, with focus on a single tendinopathy, limited range of outcome measures (e.g. pain and function) and restriction to as homogenous an intervention categorisation as possible. As a result, the number of studies included in previous reviews has been limited to between six ${ }^{21}$ and fifteen. ${ }^{23}$ Additionally, most previous systematic reviews have limited analyses to qualitative syntheses due to concerns regarding both statistical and clinical heterogeneity. However, more recent perspectives in evidence synthesis highlight that with complex interventions statistical heterogeneity should be expected and, as is the case with primary data, variance can present opportunities for informative explanatory analyses. ${ }^{27}$ Currently a range of approaches have been developed to best synthesise complex and heterogenous data, with statistical approaches including the use of network meta-analyses (NMA) that potentially combine with meta-regressions. ${ }^{27}$ The use of NMA is rapidly increasing in many disciplines with several potential advantages including the ability to combine direct and indirect estimates of treatment effectiveness to enhance precision of estimates. ${ }^{28}$ In addition, NMAs may be most effective in areas where there are multiple common treatment options, and an overall hierarchy is unclear. Here NMAs are also particularly suited to assist in creating treatment hierarchies where certain important treatment options are rarely compared directly. When combined with Bayesian methods, therapies can be separated into relatively broad treatment classes or more specific treatments and in both scenarios ranking used to quantify the probability that a specific option is most effective for a given outcome. Where treatments provide similar levels of effectiveness, probability values will be similar, and where there is clear evidence of superiority this should be evident and therefore informative for practitioners. At present there has been limited attempts to conduct NMAs within tendinopathy, with previous studies of conservative treatments primarily limited to Achilles Tendinopathy. ${ }^{29,30}$ Comparing 42 treatments and 10 treatment classes across 29 studies, van der Vlist et al. ${ }^{29}$ identified strong evidence that all treatment classes were superior to waitand-see for midportion Achilles tendinopathy, but found no evidence of clinically relevant differences in the effectiveness between active treatments at either 3 or 12 -months follow-up. Of the 65 treatments included in the trials, 40 of these comprised exercise therapies and given the associated low costs and few harms, van der Vlist et al. ${ }^{29}$ proposed that clinicians should consider at least starting treatment with exercise therapies. The authors identified that the relatively low number of studies included in the review limited the analyses as many of the treatments were not connected to the network and low statistical power negated attempts to explore heterogeneity. ${ }^{29}$ Given the extensive use and initial support for exercise therapies across the tendinopathy literature, and the lack of previous attempts to quantitatively synthesise large amounts of effectiveness data across multiple tendinopathy types, the following systematic review and meta-analysis will be conducted. Network structures will be used to compare exercise treatments and treatment classes in attempts to identify a treatment hierarchy. Additionally, the large amount of data synthesised will be used to explore relevant factors that may explain statistical heterogeneity. 


\subsection{Inclusion criteria}

This review is part of a project funded by the National Institute for Health Research (NIHR); Health Technology Assessment (HTA) 129388 Exercise therapy for the treatment of tendinopathies. The inclusion criteria are influenced by the project aims, the results of our initial scoping review mapping the exercise and tendinopathy literature 31 as well as stakeholder workshops.

\section{Participants}

This review will include people of any age or gender with a diagnosis of tendinopathy of any severity or duration and at any anatomical location. Studies that include participants with tendinopathy in the absence of full thickness or large tears, will be included. Groups where the tear size cannot be determined will also be excluded as these require different management approaches. We will accept trial authors' diagnoses where a clearly verifiable group of clinical features is reported including: pathognomonic location of pain; a symptom altering response to applied load and/or stretch, with there being a specific test for most tendinopathies; strategies to rule out differential diagnoses; ultrasound or magnetic resonance imaging confirmation of structural change. Studies with mixed groups will have data included where there is clear reporting of the tendinopathic group, or they make up $>90 \%$ of the investigated cohort. Our definition of tendinopathy therefore includes tendinopathies such as PTTD (posterior tibial tendon dysfunction), tibialis posterior tendinopathy, peroneal tendinopathy, and GTPS (greater trochanteric pain syndrome). However, it excludes plantar heel pain as this condition may respond differently to exercise therapy and could potentially confound the review findings.

\section{Intervention}

The health technology being assessed is exercise therapy for the treatment of any tendinopathy. Exercise therapies considered for inclusion will comprise five treatment classes: 1) resistance; 2) plyometric; 3) vibration; 4) flexibility and 5) proprioception (see appendix I for definitions). To enable more detailed comparisons, individual treatments will also be defined by sub-categorising resistance, flexibility and proprioception treatment classes (see appendix I). Interventions combining exercise with other active therapies (e.g. laser, shockwave, manual therapy or injection) will not be included. Exercise therapy may be delivered in a range of settings (e.g. primary care, secondary care, community, people's homes) by a range of health or exercise professionals (e.g. physiotherapists, strength \& conditioning coaches, personal trainers) or support workers, and may be supervised or unsupervised (i.e. self-management). No restrictions will be placed on these factors for inclusion.

To be included in the review, studies are required to report sufficient information regarding the exercise intervention to enable appropriate identification of treatment duration, treatment class, treatment subcategorisation and exercise dose. In clinical settings it has been recommended that exercise dose is determined by duration, frequency, and intensity; where duration reflects the amount of time accrued in a single exercise session, frequency captures the number of exercise sessions over periods such as a week, and exercise intensity is defined either in absolute terms (such as the metabolic cost of an exercise session), or in relative terms (such as the performance of a given activity as a function of some percentage of measurable maximum capacity. To be included in the review, studies are required to provide sufficient information to describe at least two of the Swinton, Shim, Pavlova, Moss, Maclean, Greig, Parkinson, Morrissey, Alexander, Cooper (2021) 
DOI | SportsRxiv Preprint version 1

three parameters describing exercise dose. Where sufficient information is not presented in the main text of a study, a search will be made of the publishers' website to check for supplementary files that may include relevant information.

Comparator

The review will include studies that compare at least two different exercise treatment classes or at least two different exercise treatments (defined in appendix I) to enable calculation of study pairwise effect sizes.

Outcomes

Based on the results of our initial scoping review and subsequent stake holder workshops we will include outcomes that assess ten domains: 1) Disability; 2) Physical function capacity; 3) Pain on loading/activity; 4) Pain over a specified time; 5) Pain without further specification 6) Patient rating overall condition; 7) Participation; 8) Quality of life; 9) Adverse effects/events; and 10) Range of motion (for studies investigating rotator cuff tendinopathy only). Definitions for each domain and example measurement tools are presented in appendix II.

Types of studies

We will include randomized controlled trials and non-randomized controlled trials where at least two intervention arms include different exercise treatments or treatment classes.

\section{Context}

The context will include primary care, secondary care or community locations in any developed nation (defined as the top 62 countries in the Human Development Index at the time of protocol development ${ }^{32}$ for the findings to be relevant to the UK context.

\subsection{Exclusion criteria}

We will exclude self-described pilot studies and non-intervention studies where the purpose of the research is to investigate the acute effects of exercise on physiological or biomechanical variables such as pain, collagen turnover or mechanical properties of tendons.

\subsection{Methods}

The review will be conducted according to the PRISMA extension statement for reporting of systematic reviews incorporating NMAs of health care interventions ${ }^{33}$ and the recent GRADE approach to drawing conclusions from NMA using a minimally contextualised framework. ${ }^{34}$ 
DOI | SportsRxiv Preprint version 1

\section{Search strategy}

The search strategy used for this study was part of a larger search conducted to scope the entire tendinopathy and exercise therapy research base. The search comprised three steps; Firstly, a limited search of MEDLINE and CINAHL using initial keywords (MH tendinopathy OR TX tendin* OR TX tendon*) AND (MH exercise OR TX exercis*) was conducted with analysis of the text words in the titles/abstracts and those used to describe articles to develop a full search strategy. Secondly, the full search strategy was adapted to each database and applied systematically to: MEDLINE, CINAHL, AMED, EMBase, SPORTDiscus, Cochrane library (Controlled trials, Systematic reviews), JBI Evidence Synthesis, PEDRo, and Epistemonikos (a full search strategy for MEDLINE is presented in appendix III). The following trial registries were also searched: ClinicalTrials.gov, ISRCTN Registry, The Research Registry, EU-CTR (European Union Clinical trials Registry), ANZCTR (Australia and New Zealand Clinical trials Registry). Finally, the third step involved conducting a search of cited and citing articles using Scopus and hand-searching a total of 130 systematic reviews that were identified to include information relevant to exercise therapy and tendinopathy. No limit was placed on language, with research studies published in languages other than English translated via Google Translate or via international collaborations of the review team members. Searches were initiated from 1998 as (i) the heavy load eccentric calf-training protocol for Achilles tendinosis by Alfredsson et al ${ }^{35}$ was published in 1998 and may be considered seminal work in the field of tendinopathy, and (ii) there has been a proliferation of research on exercise interventions for tendinopathies post 1998.

\section{Study selection}

Proquest ${ }^{\circledR}$ Refworks will be used to manage references and remove duplicates, before importing to Covidence (Melbourne, Australia) to facilitate screening. Two levels of screening will be conducted. First all titles/abstracts will be reviewed, independently, by two members of the research team. Conflicts will be resolved by discussion or by input from a third reviewer. Full-text copies of all studies included at title/abstract screening stage will be retrieved and these will also be screened independently by two members of the research team with conflicts resolved in the same way.

\section{Data extraction}

Data will be extracted independently by 8 members of the review team (PS/KC/LA/RM/LG/EP/JS/AP) into pre-piloted excel sheets. Data will be independently coded as described in the accompanying codebook (appendix IV). To quantify reliability, 10\% of studies will be selected at random and extraction completed in duplicate. Reliability will be quantified using Cohens $\mathrm{K}$ statistic ${ }^{36}$ for categorical variables and percentage agreement for continuous variables.

Risk of bias assessment

We will use the Cochrane Collaboration's Risk of Bias tool ${ }^{37}$ and all five domains: 1) selection bias; 2) performance bias; 3) detection bias; 4) attrition bias; and 5) reporting bias, to assess risk of bias for all included RCTs. For non-random designs, we will use the ROBINS-I tool 38 and all seven domains: 1) bias due to 
DOI | SportsRxiv Preprint version 1

confounding; 2) bias in selection of participants into the study; 3) bias in classification of interventions; 4) bias due to deviations form intended interventions; and 5) bias due to missing data; 6) bias in measurement of outcomes; and 7) bias in selection of the reported. An overall risk of bias judgement will be made for each outcome and time point as either 'low risk', 'some concerns' or 'high risk' of bias. A single assessment will be made by a reviewer from the team with comments saved to justify selection for each signalling question. To quantify reliability, $10 \%$ of studies will be selected at random and extraction completed in duplicate.

\section{Statistical analysis}

We will fit treatment-level and class-level Bayesian models. Pairwise effect sizes will be calculated with standardised mean differences $\left(\mathrm{SMD}_{\mathrm{pre}}\right)$ for continuous outcomes and proportional odds models used for binary outcomes. Initially, direct pair-wise comparisons will be estimated. We will then combine direct and indirect comparisons using NMA and hierarchical NMA if possible. ${ }^{39}$ Outcomes will be analysed separately according to short ( $\leq 12$ weeks), medium (13-52 weeks) and long (>52) time frames. Following the GRADE approach for presentation and interpretation of results, we will select a reference intervention defined as the most connected node in the network. To maintain a minimally contextualised framework, we will select a no effect threshold and move any treatment or treatment class above or below the reference if $95 \%$ credible intervals do not span the threshold. Second classifications will then be made based on comparisons with treatment or treatment classes moved relative to the reference. In each of the classifications, treatment or treatment classes will be separated into: 1) moderate to high certainty; and 2) low to very low certainty based on risk of bias, inconsistency and indirectness. ${ }^{40}$ Inconsistency will be assessed using model-based methods and comparison of residual deviance and the deviance information criterion). ${ }^{41}$ Finally, consistency of the treatment and treatment class hierarchies created in previous steps will be assessed by examining pairwise comparisons not previously used. Sources of statistical heterogeneity will only be explored in cases where there are 10 or more trials available per comparison. ${ }^{29}$ 


\section{References}

1. Hopkins C, Fu SC, Chua E, Hu X, Rolf C, Mattila VM, et al. Critical review on the socio-economic impact of tendinopathy. Asia Pac J Sports Med Arthrosc Rehabil Technol. 2016; 4: 9-20.

2. Lewis J. Rotator cuff related shoulder pain: Assessment, management and uncertainties. Man Ther. 2016; 23:57-68.

3. Alizadehkhaiyat O, Fisher AC, Kemp GJ. Pain, functional disability and psychologic status in tennis elbow. Clin J Pain. 2007; 23(6): 482-489.

4. Seil R, Litzenburger H, Kohn D. Arthroscopic treatment of chronically painful calcifying tendinitis of the supraspinatus tendon. Arthroscopy. 2006; 22(5): 521-527.

5. Comin J, Malliaras P, Baquie P, Barbour T, Connell D. Return to competitive play after hamstring injuries involving disruption of the central tendon. Am J Sports Med. 2013; 41(1): 111-115.

6. Fu FH, Wang JH-C, Rothrauff BB. BMJ Best Practice Tendinopathy [Internet]. London: BMJ; 2019 [cited 2019 Jul 20]. Available from:

https://bestpractice.bmj.com/topics/en-gb/582

7. Albers IS, Zwerver J, Diercks RL, Dekker JH, Van den Akker-Scheek I. Incidence and prevalence of lower extremity tendinopathy in a Dutch general practice population: a cross sectional study. BMC Musculoskelet Disord. 2016; 17:16.

8. Littlewood C, May S, Walters S. Epidemiology of Rotator Cuff Tendinopathy: A Systematic Review. Shoulder \& Elbow. 2013; 5(4): 256-265.

9. Shiri R, Viikari-Juntura E, Varonen H, Heliövaara M. Prevalence and determinants of lateral and medial epicondylitis: A population study. Am J Epidemiol. 2006; 164(11): 1065-1074.

10. Vos T. Years lived with disability (ylds) for 1160 sequelae of 289 diseases and injuries 1990-2010: a systematic analysis for the global burden of disease study 2010. Lancet. 2012; 380(9859):2163-2196.

11. Kinge JM, Knudsen AK, Skirbekk V, Vollset SE. Musculoskeletal disorders in Norway: prevalence of chronicity and use of primary and specialist health care services. BMC Musculoskelet Disord. 2015;16(1):75.

12. Abat F, Alfredson H, Cucciarini M, Madry H, Marmott A, Mouton C, et al. Current trends in tendinopathy: consensus of the ESSKA basic science committee. Part I: biology, biomechanics, anatomy and an exercise-based approach. J Exp Orthop. 2017; 4:18.

13. Alfredson H. The chronic painful Achilles and patellar tendon: Research on basic biology and treatment. Scand J Med Sci Sports. 2005; 15(4): 252-259.

14. O'Neill S, Watson P, Barry S. Why are eccentric exercises effective for Achilles tendinopathy? Int J Sports Phys Ther. 2015; 10(4): 552-62.
15. Lim HY, Wong SH. Effects of isometric, eccentric, or heavy slow resistance exercises on pain and function in individuals with patellar tendinopathy: A systematic review. Physiother Res Int. 2018 Oct;23(4):e1721.

16. Rio E, et al Tendon neuroplastic training: Changing the way we think about tendon rehabilitation: A narrative review Br J Sports Med. 2016; 50(4): 209-215.

17. Worsley et al. Motor control retraining exercises for shoulder impingement: effects on function, muscle activation, and biomechanics in young adults. J Shoulder Elbow Surg. 2013; 22(4): e11-19.

18. Ager et al. The effectiveness of an upper extremity neuromuscular training program on the shoulder function of military members with a rotator cuff tendinopathy: A Pilot Randomized Controlled Trial. Mil Med. 2019; 184(56): e385-e393.

19. Lewis J, McCreesh K, Roy JS, Ginn K. Rotator Cuff Tendinopathy: Navigating the Diagnosis-Management Conundrum. J Orthop Sports Phys Ther. 2015 Nov;45(11):923-37.

20. Dimitrios S. Exercise for tendinopathy. World J Methodol. 2015; 5(2): 51-54.

21. Shire et al. Specific or general exercise strategy for subacromial impingement syndrome-does it matter? A systematic literature review and meta analysis. BMC Musculoskelet Disord. 2017; 18(1): 158.

22. Gutiérrz-Espinoza et al. Effect of supervised physiotherapy versus home exercise program in patients with subacromial impingement syndrome: A systematic review and meta-analysis. Phys Ther Sport. 2020; 41: 34-42.

23. Lim HY, Wong SH. Effects of isometric, eccentric, or heavy slow resistance exercises on pain and function in individuals with patellar tendinopathy: A systematic review. Physiother Res Int. 2018 Oct;23(4):e1721.

24. Naunton J, Street G, Littlewood C, Haines T, Malliaras $P$. Effectiveness of progressive and resisted and nonprogressive or non-resisted exercise in rotator cuff related shoulder pain: A systematic review and meta-analysis of randomized controlled trials. Clin Reahbil. 2020; 34(9): 1198-1216

25. Chen Z, Baker NA. Effectiveness of eccentric strengthening in the treatment of lateral elbow tendinopathy: A systematic review with meta-analysis. J Hand Ther. 2021; 34(1): 18-28.

26. Malliaras P, Barton CJ, Reeves ND, Langberg H. Achilles and patellar tendinopathy loading programmes: A systematic review comparing clinical outcomes and identifying potential mechanisms for effectiveness. Sports Med. 2013; 43(4): 267-286.

27. Lorenc et al. Meta-analysis, complexity, and heterogeneity: A qualitative interview study of researchers' methodological values and practices. Syst Rev. 2016; 5(1): 192. 
28. Veer ET, van Oijen MGH, van Laarhoven HWM. The use of (network) meta-analysis in clinical oncology. Front Oncol. 2019; 9: 822.

29. van der Vlist et al. Which treatment is most effective for patients with Achilles tendinopathy? A living systematic review with network meta-analysis of 29 randomised controlled trials. Br J Sports Med. 2021; 55(5): 249-256.

30. Rhim HC, Kim MS, Choi S, Tenforde AS. Comparative efficacy and tolerability of nonsurgical therapies for the treatment of midportion Achilles tendinopathy. A systematic review with network meta-analysis. Orthop J Sports Med. 2020; 8(7): 2325967120930567.

31. Alexander et al. Exercise therapy for the treatment of tendinopathies: A scoping review protocol. JBI Evid Synth. 2021; doi: 10.11124/JBIES-20-00175.

32. United Nations Development Programme-Human Development Reports. Human development index: Table 1 human development index and its components 2020; Retrieved from: http://hdrundporg/en/composite/HDI.

33. Hutton et al. The PRISMA extension statement for reporting of systematic reviews incorporating network meta-analyses of health care interventions: Checklists and explanations. Ann Intern Med. 2015; 26(11): 777-784.

34. Brignardello-Petersen et al. Grade approach to drawing conclusions from a network meta-analysis using a minimally contextualised framework. BMJ. 2020; 371:m3900.
35. Alfredson H, Pietilä T, Jonsson P, Lorentzon R. Heavyload eccentric calf muscle training for the treatment of chronic Achilles tendinosis. Am J Sports Med. 1998; 26(3): 360-366.

36. Cohen J. Weighted kappa: nominal scale agreement with provision for scaled disagreement or partial credit. Psychol Bull. 1968; 70: 213-220.

37. Higgins et al. The Cochrane Collaboration's tool for assessing risk of bias in randomised trials. BMJ. 2011; 343:d5928.

38. Sterne et al. ROBINS-I: A tool for assessing risk of bias in non-randomised studies of interventions. BMJ. 2016; 355:14919.

39. Owen RK, Tincello DG, Keith RA. Network metaanalysis: development of a three-level hierarchical modelling approach incorporating dose-related constraints. Value Health. 2015; 18(1): 116-126.

40. Guyatt et al. GRADE: An emerging consensus on rating quality of evidence and strength of recommendations. BMJ. 2008; 336:924.

41. Dias et al. Evidence synthesis for decision making 4: Inconsistency in networks of evidence based on randomized controlled trials. Med Decis Making. 2013; 33(5): 641-656. 
Appendix I: Definitions use to define exercise treatments and treatment classes.

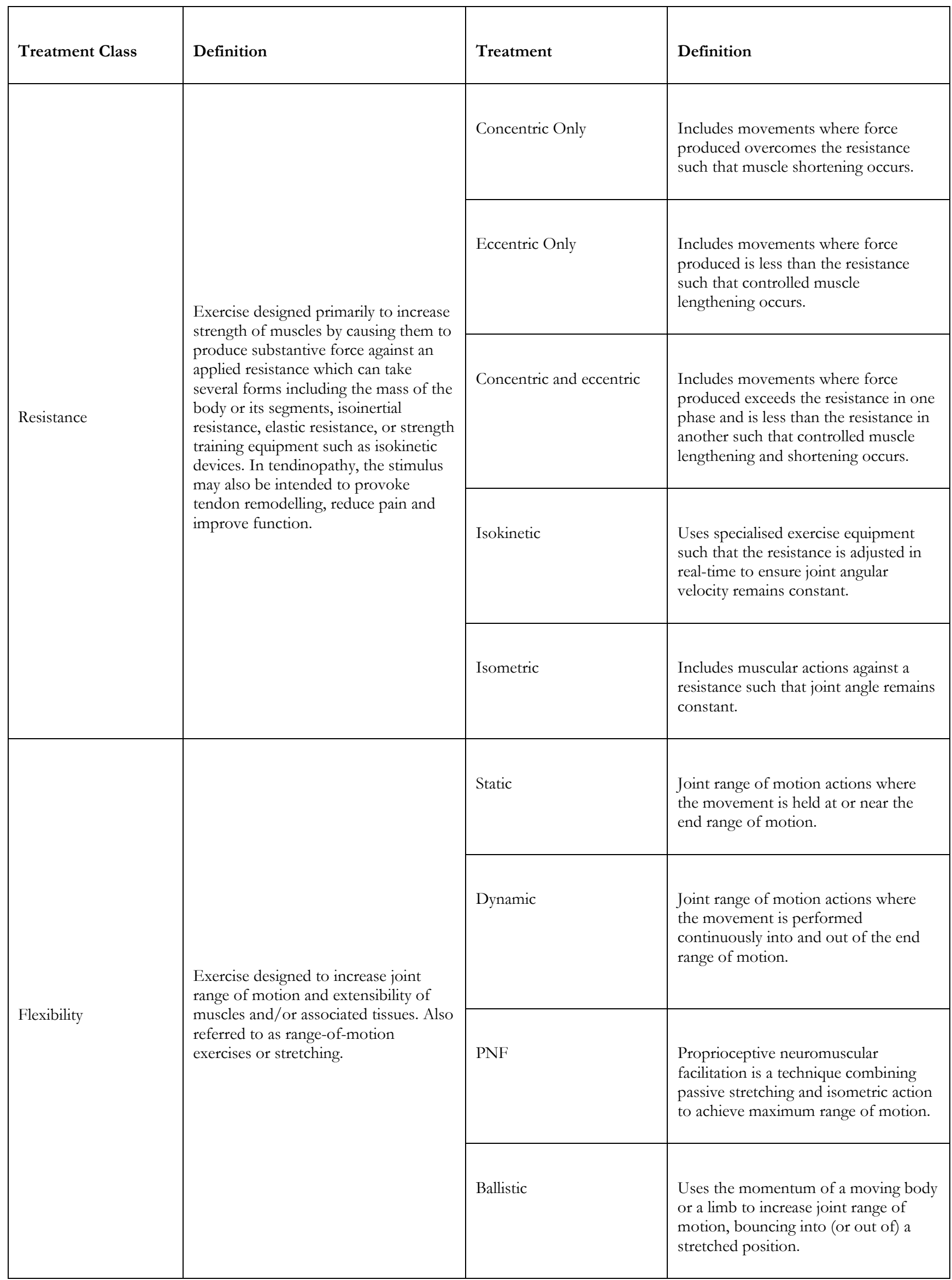




\begin{tabular}{|c|c|c|c|}
\hline \multirow{3}{*}{ Proprioception } & \multirow{3}{*}{$\begin{array}{l}\text { Exercise designed to enhance the } \\
\text { sensation of the joint relative to body } \\
\text { position and movement, sense of force, } \\
\text { and to encourage muscular stabilisation } \\
\text { of the joint in the absence of external } \\
\text { stabilising devices e.g. ankle brace. }\end{array}$} & $\begin{array}{l}\text { Sense of joint position and } \\
\text { force }\end{array}$ & $\begin{array}{l}\text { Exercise aimed at enhancing the ability } \\
\text { to perceive joint position and force } \\
\text { with minimal external cues. }\end{array}$ \\
\hline & & Balance & $\begin{array}{l}\text { Includes exercise that require the } \\
\text { person to keep or return the } \\
\text { displacement of centre of gravity over } \\
\text { the base of support through various } \\
\text { environmental conditions and changes } \\
\text { in body position. }\end{array}$ \\
\hline & & $\begin{array}{l}\text { Movement pattern } \\
\text { retraining }\end{array}$ & $\begin{array}{l}\text { Exercise aimed at re-education of } \\
\text { motor control and movement patterns } \\
\text { that may involve specific retraining of } \\
\text { under- or over-active muscles and } \\
\text { alteration of kinematic rotation }+- \\
\text { translation timing between body } \\
\text { segments. May also be termed motor } \\
\text { control or stabilisation. }\end{array}$ \\
\hline Plyometric & $\begin{array}{l}\text { Exercise where a resistance is } \\
\text { overcome by a muscle rapidly } \\
\text { stretching then shortening }\end{array}$ & Plyometric & $\begin{array}{l}\text { Exercise where a resistance is } \\
\text { overcome by a muscle rapidly } \\
\text { stretching then shortening. }\end{array}$ \\
\hline Vibration & $\begin{array}{l}\text { Exercise where body segments are held } \\
\text { stationary or actively displaced as per } \\
\text { definitions for other treatment classes } \\
\text { whilst applying a rapid oscillating } \\
\text { resistance }\end{array}$ & Vibration & $\begin{array}{l}\text { Exercise where body segments are held } \\
\text { stationary or actively displaced as per } \\
\text { definitions for other treatment classes } \\
\text { whilst applying a rapid oscillating } \\
\text { resistance }\end{array}$ \\
\hline
\end{tabular}




\section{Appendix II: Outcome domains and example outcomes included in review.}

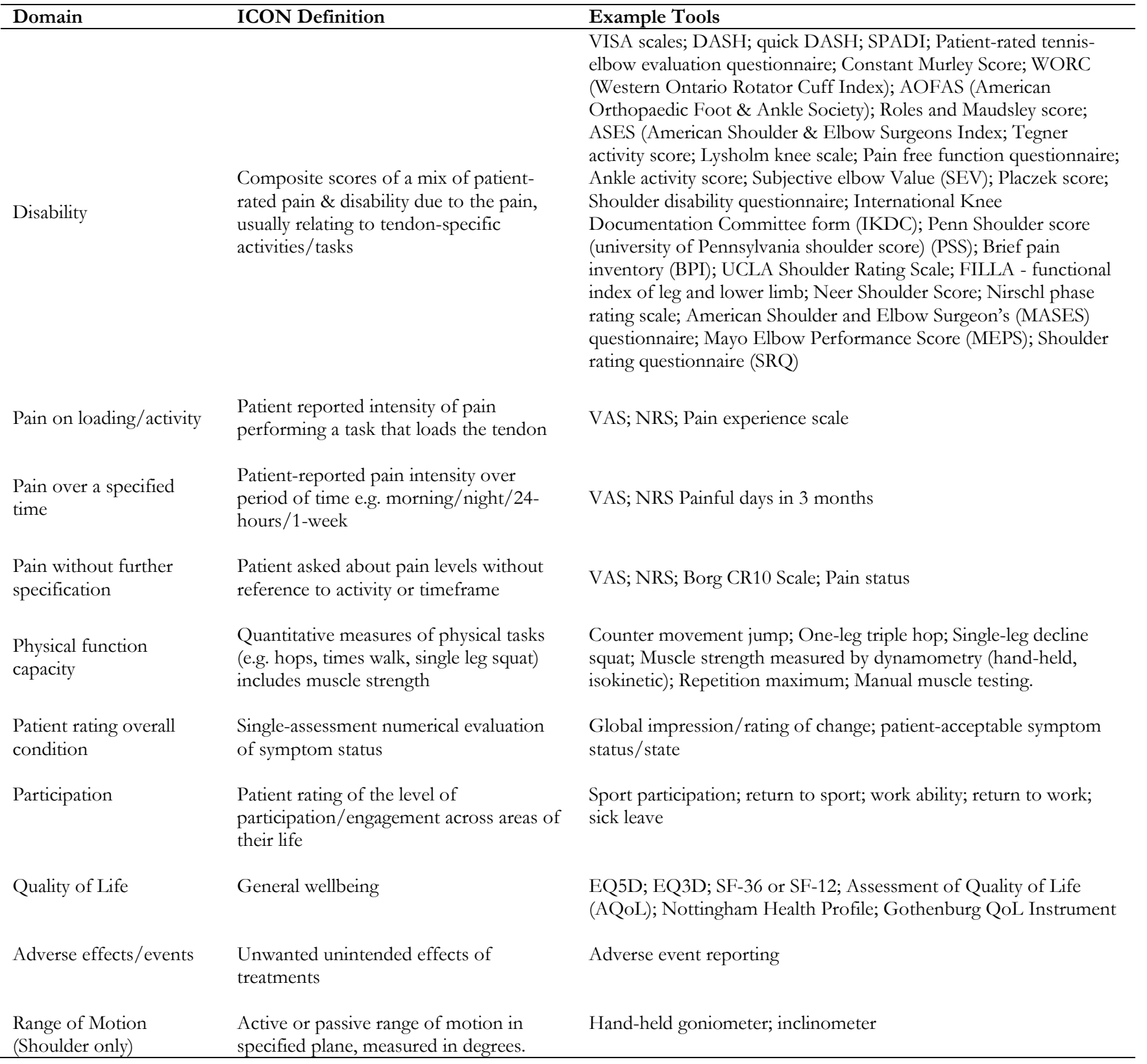


DOI | SportsR $x$ iv Preprint version 1

\section{Appendix III: Search strategy}

MEDLINE (EBSCoHost)

Search conducted on 27 April, 2020

\begin{tabular}{|l|l|l|}
\hline Search & Query & $\begin{array}{l}\text { Records } \\
\text { retrieved }\end{array}$ \\
\hline$\# 1$ & $\begin{array}{l}\text { MH exercise OR AB exercis* OR MH “isometric contraction” OR MH rehabilitation OR TX } \\
\text { eccentric OR TX concentric OR TX “heavy slow resistance" OR TX isokinetic }\end{array}$ & 362,722 \\
\hline$\# 2$ & $\begin{array}{l}\text { MH tendinopathy OR MH “shoulder injuries" OR MH tendons OR MH “tendon injuries” OR } \\
\text { TX tendin* OR TX tendon* OR MH bursitis OR AB bursitis OR MH “posterior tibial tendon } \\
\text { dysfunction" OR MH “shoulder impingement syndrome” OR AB “greater trochanteric pain } \\
\text { syndrome" }\end{array}$ & 96,490 \\
\hline$\# 3$ & $\# 1$ AND \#2 & 4,363 \\
\hline Limited to 1998 to present & \\
\hline
\end{tabular}




\section{Appendix IV: Extraction codebook}

\begin{tabular}{|c|c|c|c|}
\hline \multicolumn{2}{|c|}{ Column } & \multirow{2}{*}{$\begin{array}{l}\text { Heading } \\
\text { Initials Reviewer }\end{array}$} & \multirow{2}{*}{$\begin{array}{c}\text { Description } \\
\text { Identification of individual extracting information }\end{array}$} \\
\hline \multirow{23}{*}{ 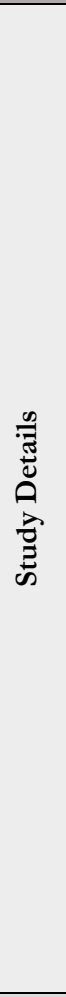 } & A & & \\
\hline & $\mathrm{B}$ & Covidence Identifier & Reference number for Covidence \\
\hline & $\mathrm{C}$ & Author & First author surname et al., \\
\hline & $\mathrm{D}$ & Year & Year of publication \\
\hline & $\mathrm{E}$ & Title & Study title \\
\hline & $\mathrm{F}$ & Country & Country where study was conducted \\
\hline & G & Journal & Journal name \\
\hline & $\mathrm{H}$ & Aims/Purpose & Study aims/purpose \\
\hline & I & Tendinopathy type & 1=Achilles; $2=$ Lateral elbow (tennis); $3=$ Patellar; $4=$ Rotator cuff $(\mathrm{SI})$ \\
\hline & $\mathrm{J}$ & Study Design & $\mathrm{RCT}=1 ;$ Quasi-experimental $=2$ \\
\hline & $\mathrm{K}$ & Age Mean & Mean age of study sample as a whole \\
\hline & $\mathrm{L}$ & Age SD & Standard deviation age of study sample as a whole \\
\hline & $\mathrm{M}$ & Baseline Total N & Total sample across all interventions measured at baseline \\
\hline & $\mathrm{N}$ & $\begin{array}{l}\text { Training Status } \\
\text { Description }\end{array}$ & Brief description of training status of study sample as a whole \\
\hline & $\mathrm{O}$ & Training Status Code & $1=$ Performance; $2=$ Sporting; 3 = Other \\
\hline & $\mathrm{P}$ & Sex & Percentage female of study sample as a whole \\
\hline & Q & BMI Mean & Mean BMI of study sample as a whole \\
\hline & $\mathrm{R}$ & BMI SD & Standard deviation of BMI of study sample as a whole \\
\hline & $\mathrm{S}$ & Symptom Severity Mean & Mean severity measure at baseline of study sample as a whole \\
\hline & $\mathrm{T}$ & Symptom Severity SD & Standard deviation of severity measure at baseline of study sample as a whole \\
\hline & $\mathrm{U}$ & $\begin{array}{l}\text { Symptom Duration } \\
\text { Mean (Months) }\end{array}$ & Mean symptom duration reported in months \\
\hline & $\mathrm{V}$ & $\begin{array}{l}\text { Symptom Duration SD } \\
\text { (Months) }\end{array}$ & Standard deviation symptom duration reported in months \\
\hline & W & Population Comments & $\begin{array}{l}\text { Any additional information relevant to the participants investigated including } \\
\text { diagnostic criteria }\end{array}$ \\
\hline \multirow{4}{*}{ 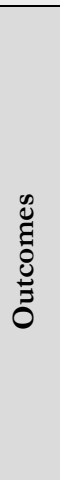 } & $\mathrm{X}$ & Outcome Category & $\begin{array}{c}1=\text { Disability; } 2 \text { = Pain on loading/activity; } 3=\text { Pain over a specified time; } 4= \\
\text { Pain without further specification; } 5=\text { Physical function capacity; } 6=\text { Patient } \\
\text { rating overall condition; 7) Participation; 8) Quality of life; 9) Adverse } \\
\text { effects/events; 10) Range of motion }\end{array}$ \\
\hline & & Outcome Tool & Description of outcome tool \\
\hline & $\mathrm{Z}$ & Reflection & $\begin{array}{c}1=\text { Increase in outcome indicates positive treatment; }-1=\text { Decrease in outcome } \\
\text { indicates positive treatment }\end{array}$ \\
\hline & $\mathrm{AA}$ & $\begin{array}{l}\text { Measurement Time } \\
\text { (Weeks) }\end{array}$ & Time of measurement in weeks \\
\hline \multirow{8}{*}{ 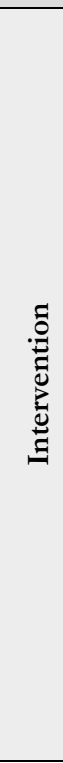 } & $\mathrm{AB}$ & $\begin{array}{l}\text { Dominant Treatment } \\
\text { Class }\end{array}$ & $\begin{array}{l}\text { Only one dominant theme to be selected } \\
1=\text { Resistance; } 2=\text { Plyometric; } 3=\text { Vibration; } 4=\text { Flexibility; } 5=\text { Proprioception }\end{array}$ \\
\hline & $\mathrm{AC}$ & Total Treatment class & $\begin{array}{l}\text { Multiple themes to be selected as required } \\
1=\text { Resistance; } 2=\text { Plyometric; } 3=\text { Vibration; } 4=\text { Flexibility; } 5=\text { Proprioception }\end{array}$ \\
\hline & $\mathrm{AD}$ & Dominant Treatment & $\begin{array}{c}\text { Only one dominant theme to be selected } \\
1=\text { Concentric only; } 2=\text { Eccentric only; } 3=\text { Concentric and eccentric; } 4= \\
\text { Isokinetic; } 5=\text { Isometric; } 6=\text { Static; } 7=\text { Dynamic; } 8=\text { PNF; } 9=\text { Ballistic; } 10= \\
\text { Joint position \& force; } 11=\text { Balance; } 12=\text { Movement pattern retraining; } 13= \\
\text { Plyometric; } 14=\text { Vibration }\end{array}$ \\
\hline & $\mathrm{AE}$ & Total Treatment & $\begin{array}{c}\text { Multiple themes to be selected as required } \\
1=\text { Concentric only; } 2=\text { Eccentric only; } 3=\text { Concentric and eccentric; } 4= \\
\text { Isokinetic; } 5=\text { Isometric; } 6=\text { Static; } 7=\text { Dynamic; } 8=\text { PNF; } 9=\text { Ballistic; } 10= \\
\text { Joint position \& force; } 11=\text { Balance; } 12=\text { Movement pattern retraining; } 13= \\
\text { Plyometric; } 14=\text { Vibration }\end{array}$ \\
\hline & $\mathrm{AF}$ & Intervention $\mathrm{N}$ & $\begin{array}{l}\text { Intervention sample size at specified time } \\
\end{array}$ \\
\hline & AG & $\begin{array}{l}\text { Intervention Total } \\
\text { Duration }\end{array}$ & Total duration of exercise intervention in weeks \\
\hline & $\mathrm{AH}$ & $\begin{array}{l}\text { Intervention Adherence } \\
\%\end{array}$ & Reporting of adherence to exercise (reported as a percentage) if applicable \\
\hline & AI & Intervention Location & $\begin{array}{l}\text { Location exercise was performed } \\
1=\text { Home; } 2=\text { Clinic; } 3=\text { Fitness facility; } 4=\mathrm{NR} ; 5=\mathrm{NA}\end{array}$ \\
\hline
\end{tabular}

Swinton, Shim, Pavlova, Moss, Maclean, Greig, Parkinson, Morrissey, Alexander, Cooper (2021) 


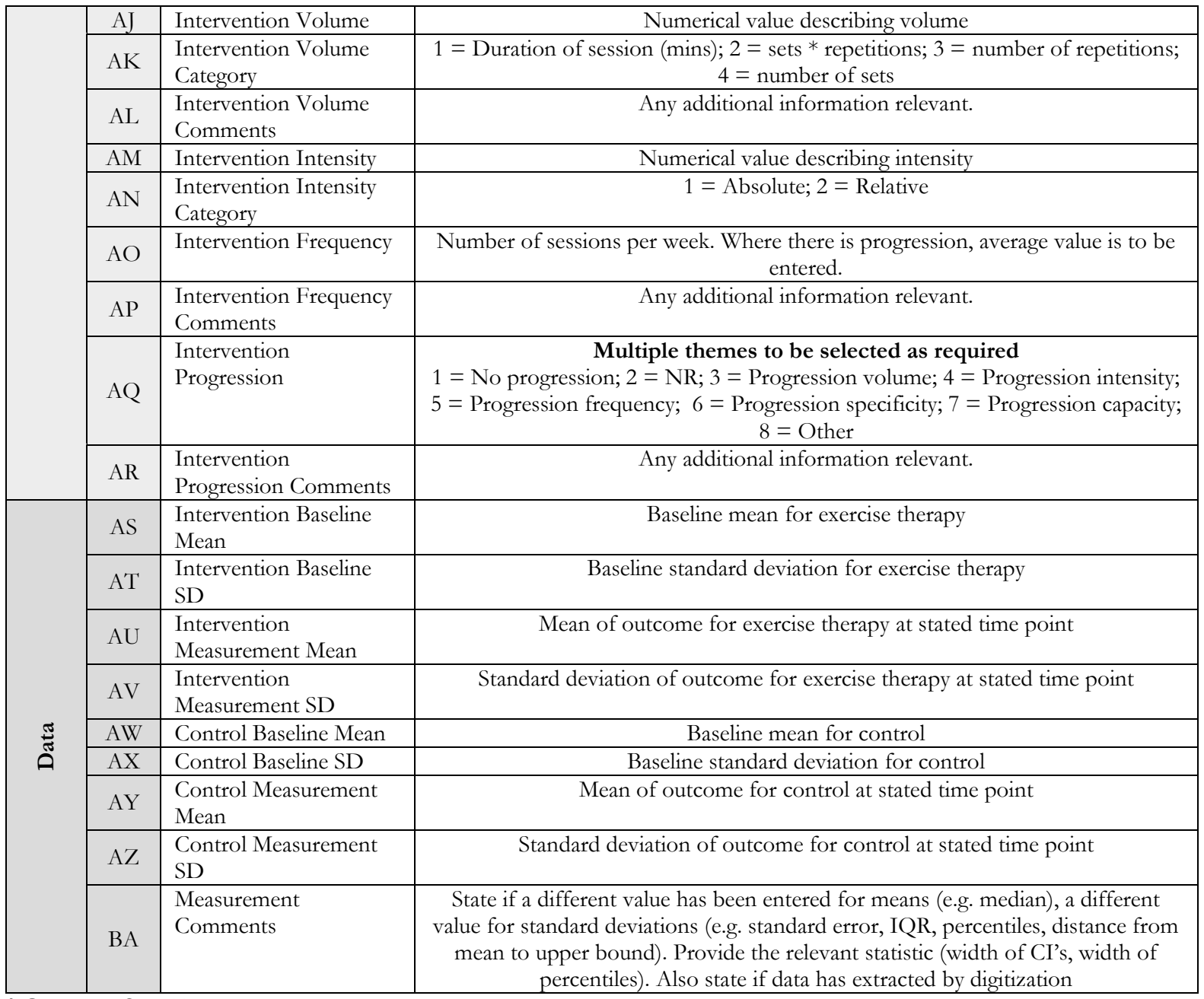

\section{* Outcome Specific}

